rates in hepatocellular carcinoma (HCC), which has been attributable to the strong immunosuppressive tumor microenvironment (TME). As a key player in the TME, myeloidderived suppressor cell (MDSC) shows potent T cell-suppressive activity that remarkably associates with poor prognosis and ICB resistance of cancer patients. While targeting MDSC can blunt $\mathrm{T}$ cell activity, a new approach is directed towards driving MDSC differentiation into antigen presentation cell crucial for $\mathrm{T}$ cell priming and activation. We have recently shown that hepatoma-intrinsic cyclin-dependent-kinase 20 (CDK20), or cell-cycle-related-kinase (CCRK) depletion diminishes MDSC-mediated immunosuppression leading to improved ICB efficacy (Gut 2018). As emerging evidence highlights the key roles of immune cell-intrinsic CDKs, we aimed to further explore the potentials of CCRK in immune cell identity.

Methods The expression profile of CCRK was determined in flow-sorted immune cells from tumor-bearing mice and HCC patients. Functional significance and molecular mechanisms of CCRK in MDSCs were conducted by gene knockdown in human blood-derived MDSCs, followed by mRNA and protein detection, qChIP-PCR and multi-colour flow cytometry. The MDSC differentiation and $\mathrm{T}$ cell suppression in tumorigenicity were validated in HCC mouse model with intratumoral MDSC injection.

Results We uncovered specific over-expression of CCRK in MDSCs but not lymphocytes from tumor-bearing mice and HCC patients. Notably, blockade of MDSC-intrinsic CCRK induced its differentiation into antigen-presenting macrophage, which amplified $\mathrm{T}$ cell responses in vitro and in vivo, resulting in reduced tumorigenicity. CCRK inhibition suppressed signal transducer and activator of transcription 3 (STAT3) signaling to revert E4-binding protein 4 (E4BP4)-dependent interleukin10 (IL-10)/IL-12 imbalance and arginase I expression, thus blunting immunosuppression.

Conclusions Our findings demonstrate that targeting myeloidintrinsic CCRK signal can amplify anti-tumor $\mathrm{T}$ cell responses. As we also showed CCRK overexpression in patient-derived MDSCs, our results not only unravel mechanistic insights in MDSC identity but also offer a novel therapeutic kinase-target for a combinational immunotherapy strategy for conferring durable eradication of solid tumors.

\section{IDDF2020-ABS-0182 A NOVEL IMMUNOSUPPRESSIVE ROLE OF CHOLESTEROL IN NAFLD-ASSOCIATED HEPATOCARCINOGENESIS}

Wenshu Tang*, Jingying Zhou, Weiqin Yang, Yu Feng, Heung Man Lee, Candice CH Lam, Anthony WH Chan, Simon KH Wong, Vincent WS Wong, Alice PS Kong, Alfred SL Cheng. The Chinese University of Hong Kong, Hong Kong

\subsection{6/gutjnl-2020-IDDF.37}

Background Hepatocellular carcinoma (HCC) is the fifth most prevalent cancer and the second leading cause of cancer deaths worldwide. Driven by the epidemics of obesity and diabetes, it is anticipated that non-alcoholic fatty liver disease (NAFLD) will become the most important cause of HCC. Emerging evidence suggests that metabolic and immune dysfunction are key features of NAFLD-associated HCC, my research aim to investigate the metabolic-immune dysregulation underlying NAFLD-HCC development. Here we show that aberrant cholesterol accumulation in the liver predisposes cancer development by reprogramming the immunosurveillance microenvironment.

Methods Comprehensive immune profiling in a high-fat highcarbohydrate diet-induced NAFLD murine model was performed.

Results Our results revealed a specific reduction of cytolytic natural killer $\mathrm{T}$ (NKT) cells in the liver, which was negatively correlated with the elevated cholesterol level. Cholesterol-lowering drug Rosuvastatin reduced cholesterol level and restored NKT cell proportion and function, which subsequently suppressed the growth of orthotopically-implanted HCC tumor. Inhibition of cholesterol synthesis by an mTORC1/C2 dual kinase inhibitor Vistusertib (AZD2014), or lentiviral-mediated suppression of the mTOR pathway also showed similar effects. Notably, NKT inactivation by a specific CD1d receptor-targeting antibody abolished the anti-tumorigenic effects of both Rosuvastatin and Vistusertib, thus underscoring the casual role of NKT in cholesterol-mediated hepatocarcinogenesis. We also showed a reduction of NKT cells and its negative correlation with cholesterol elevation in obese patients undergone bariatric surgery.

Conclusions Our study elucidates a new immunosuppressive role of cholesterol in establishing a pro-tumorigenic microenvironment. These findings explain why cholesterol-lowering drugs may reduce cancer incidence and provide new therapeutic strategies of revitalizing the immunosurveillant NKT cells for the intervention of NAFLD-associated HCC.

This project is supported by the University Grants Committee through the Collaborative Research Fund (C4045-18W), General Research Fund (14108219, 14105419), the Li Ka Shing Foundation and the Terry Fox Foundation and the AstraZeneca Preclinical Oncology Research Program (2017).

\section{IDDF2020-ABS-0201 TARGETING HEPATOMA-INTRINSIC PPAR $\gamma$ SIGNALING OVERCOMES IMMUNE CHECKPOINT THERAPY RESISTANCE BY INFLAMING THE TUMOR MICROENVIRONMENT}

Zhewen Xiong*, Stephen Chan, Jingying Zhou, Jianquan Cao, Joaquim SL Vong, Xuezhen Zeng, Yalin Tu, Yu Feng, Kevin Yip, Joseph JY Sung, Alfred Sze-Lok Cheng. The Chinese University of Hong Kong, Hong Kong

\subsection{6/gutjnl-2020-IDDF.38}

Background Immune-checkpoint blockade (ICB) therapies by antibodies against programmed death 1 (PD1)/PD1 ligand 1 (PD-L1) axis have revolutionized the treatment paradigm for cancer. Although subsets of people exhibit durable responses, ICB resistance has increasingly been observed, especially in hepatocellular carcinoma (HCC). Here we utilized a single-cell RNA-sequencing (scRNA-seq) approach to elucidate the tumorintrinsic mechanism underlying tumor immunosuppression and ICB resistance.

Methods We first recapitulated the clinical outcome of ICB resistance via repeated cycles of in vivo selection in orthotopic murine models of HCC. To investigate the tumor cell-extrinsic resistant factors, the myeloid and lymphoid immune populations were profiled by multi-color flow cytometry. To dissect hepatoma-intrinsic resistant signatures, we performed scRNAseq from anti-PD-L1-treated tumors generated from parental or PD-L1R Hepa1-6 cells. The anti-tumor efficacy and immunophenotype of combined therapy with anti-PD-L1 antibody 
and peroxisome proliferator-activated receptor gamma (PPAR $\gamma$ ) antagonist T0070907 were further determined. To demonstrate the clinical relevance of PPAR $\gamma$, we performed scRNA-seq analysis of tumor biopsies from advanced HCC patients who received anti-PD-1 treatment.

Results We successfully established anti-PD-L1-resistance models, which were accompanied with lower $\mathrm{CD} 8^{+} \mathrm{T}$ cells and $\mathrm{T}$ helper $1\left(\mathrm{~T}_{\mathrm{H}} 1\right)$ cells but higher exhausted $\mathrm{T}$ cells and myeloid-derived suppressor cells (MDSCs). Integrative gene expression analysis showed significant enrichment of PPAR $\gamma$ signaling in PD-L1R tumor cells. Importantly, T0070907 overcame ICB resistance in HCC, which was accompanied with enhanced cytolytic activity and reduced $\mathrm{T}$ cell exhaustion and decreased infiltration of MDSCs. Notably, scRNA-seq profiles of human biopsies uncovered adaptive upregulation of tumor-cell intrinsic PPAR $\gamma$ and re-shaping of T cell exhaustion in non-responders upon anti-PD-1 therapy.

Conclusions Taken together, hepatoma-intrinsic PPAR $\gamma$ activation might be associated with immune evasion and ICB resistance. Pharmacological inhibition of PPAR $\gamma$ sensitized tumors to anti-PD-L1 therapy, thus representing a promising strategy to overcome ICB resistance.

\section{IDDF2020-ABS-0209 ASIAN PREVALENT ALLELE AT ABCB5 SNP RS10254317 ASSOCIATES WITH HEPATOCELLULAR CARCINOMA (HCC) RISK AND ADVERSE CLINICAL OUTCOMES}

${ }^{1}$ Philip Chun Yeung*, ${ }^{1}$ Charing Ching-Ning Chong, ${ }^{2}$ Tan To Cheung, ${ }^{1}$ Kelvin Kwok Chai Ng, ${ }^{1}$ Paul Bo San Lai, 'Siu Tim Cheung. 'The Chinese University of Hong Kong, Hong Kong;

${ }^{2}$ The University of Hong Kong, Hong Kong

\subsection{6/gutjnl-2020-IDDF.39}

Background Ethnic disparities in the prevalence of hepatocellular carcinoma (HCC) continue to exist. Highest age-adjusted HCC incidence rates are recorded in East Asia, and $55 \%$ of HCC cases worldwide are from China. Meanwhile, ABCB5 has been reported to be overexpressed in HCC and associated with poor survival. To evaluate the ethnic differences, allele frequencies of ABCB5 SNP rs10254317 in the local cohort were compared with those from Chinese and Caucasian in 1000 Genomes project, and their clinical implications on HCC patients were studied.

Methods A total of 300 HCC and 300 healthy blood samples $\mathbf{9 9 . 3 \%}$ and $94.7 \%$ Chinese respectively) were prospectively collected with informed consent. All patients had been diagnosed with primary HCC and underwent partial hepatectomy. Clinicopathological information including sex, age, tumour stage and survival outcomes were collected prospectively. Genomic DNA was extracted from blood samples and SNPs were examined. For comparison, genomics data and corresponding clinical information for HCC were obtained from The Cancer Genome Atlas (TCGA). SNP allele frequencies in different populations were obtained from 1000 Genomes Browser by NCBI.

Results Allele frequencies of rs10254317 observed in healthy local cohorts (G: 0.334 vs A: 0.666 ) were comparable to Northern and Southern Chinese (CHB; G: 0.311 vs A: 0.689; CHS; G: 0.286 vs A: 0.714) in 1000 Genomes project, which were significantly different from Caucasian (GBR; G: 0.615 vs A: 0.385$)(\mathrm{p}<0.001)$. Chinese-dominant allele frequency (AA/ AG) associated with higher HCC risk (OR: 2.059, 95\%CI:

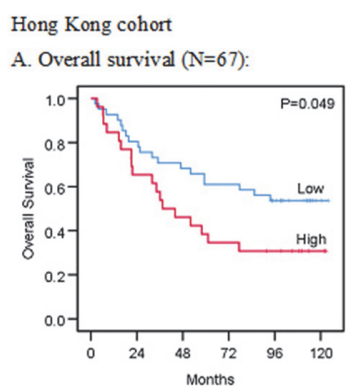

TCGA LIHC cohort

B. Overall survival - Asian $(\mathrm{N}=155)$ :

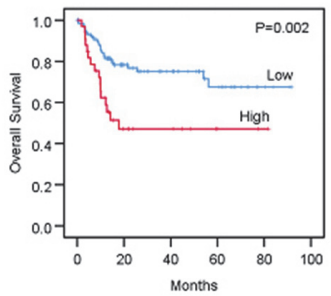

C. Overall survival - Caucasian $(\mathrm{N}=182)$

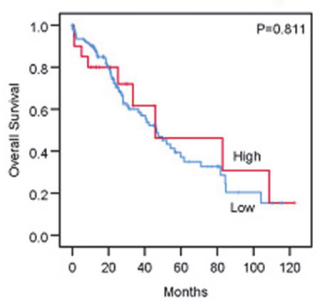

Abstract IDDF2020-ABS-0209 Figure 1 ABCB5 expression associated with poor survival outcomes in Asian HCC patients

1.16-3.67, $\mathrm{p}=0.014)$, advanced tumor stage (OR: 4.514, 95\% CI: $1.02-19.96, p=0.047)$ and presence of venous infiltration (OR: 2.864, 95\%CI: 1.00-8.18, $\mathrm{p}=0.049$ ) from local cohort. TCGA HCC dataset also revealed a disparity in HCC survival outcomes among different populations, as elevated ABCB5 expression levels associated with poor survival in Asian HCCs but not in Caucasian HCCs (figure 1).

Conclusions Dominant allele of rs10254317 in ABCB5 among Chinese associates with risk of HCC and adverse clinical outcomes in HCC patients, which may also contribute to the ethnic disparity in HCC incidence and survival outcomes. Further investigation on SNPs of HCC-related genes with ethnic disparities are warranted.

\section{IDDF2020-ABS-0215 ENHANCER REPROGRAMMING BY SELECTIVE HDAC8 INHIBITION POTENTIATES TUMOR REMISSION AND DURABLE BENEFIT BY PD-L1 BLOCKADE}

${ }^{1}$ Weiqin Yang*, ${ }^{1} Y u$ Feng, ${ }^{1}$ Jingying Zhou, ${ }^{1}$ Otto Ka Wing Cheung, ${ }^{2}$ Feng Wu, ${ }^{3}$ Zhiwu Tan, ${ }^{1}$ Liangliang Xu, ${ }^{4}$ Hanyong Sun, ${ }^{5}$ Yuan Tian, ${ }^{6}$ John Wong, ${ }^{6}$ Paul Bo San Lai, ${ }^{7}$ Stephen Lam Chan, ${ }^{2}$ Wing Hung Chan, ${ }^{8}$ Patrick Tan, ${ }^{3}$ Zhiwei Chen, ${ }^{9}$ Joseph Jao Yiu Sung, ${ }^{10}$ Kevin Yuk Lap Yip, ${ }^{2}$ Ka Fai To, ${ }^{1}$ Alfred Sze Lok Cheng. 'School of Biomedical Sciences, The Chinese University of Hong Kong, Hong Kong; ${ }^{2}$ Department of Anatomical and Cellular Pathology, The Chinese University of Hong Kong, Hong Kong; ${ }^{3}$ AIDS Institute, The University of Hong Kong, Hong Kong; ${ }^{4}$ Department of Liver Surgery, Ren Ji Hospital, School of Medicine, Shanghai Jiao Tong University, China; ${ }^{5}$ Department of Biochemistry and Molecular Biology, Shenzhen University School of Medicine, China; ${ }^{6}$ Department of Surgery, The Chinese University of Hong Kong, Hong Kong; ${ }^{7}$ Department of Clinical Oncology, The Chinese University of Hong Kong, Hong Kong; ${ }^{8}$ SingHealth Duke-NUS Institute of Precision Medicine, National Heart Centre Singapore, Singapore; ${ }^{9}$ Department of Medicine and Therapeutics, The Chinese University of Hong Kong, Hong Kong; ${ }^{10}$ Department of Computer Science and Engineering, The Chinese University of Hong Kong, Hong Kong

\subsection{6/gutjnl-2020-IDDF.40}

Background The insufficient $\mathrm{T}$ cell infiltration into noninflamed tumors such as hepatocellular carcinoma (HCC) restricts the effectiveness of immune-checkpoint blockade (ICB) to a minority of patients. Epigenetic therapy provides new opportunities to rewire cancer transcriptional programs, 